\title{
INTRINSIC VISCOSITY AND FRICTION COEFFICIENT OF PERMEABLE MACROMOLECULES IN SOLUTION
}

\author{
F.W. WIEGEL and P.F. MIJNLIEFF \\ Department of Applied Physics, Twente University of Technology, \\ Enschede, The Netherlands
}

Received 21 January 1977

\begin{abstract}
A polymer molecule in solution is treated as a porous sphere with a spherically symmetric permeability distribution. Solvent motion in and around this sphere is described by the DebijeBrinkman equation (Navier-Stokes equation and Darcy equation combined). The model allows a straightforward calculation of the frictional properties of a polymer in shear flow (intrinsic viscosity) and in translation (friction coefficient). Calculations have been carried out for a radial dependence of the permeability of the form $k(r)=K \exp \left(Q r^{2}\right)$. The calculations provide us with detailed information about the solvent flow through and around the macromolecular coil.
\end{abstract}

\section{Introduction}

In a celebrated paper Einstein ${ }^{1}$ ) calculated the viscosity of a dilute suspension of impermeable spheres. Einstein's expression for the viscosity is often used to interpret the viscosity of a solution of macromolecules in terms of an effective hydrodynamic radius. However, a macromolecule in solution cannot be represented by an impermeable sphere because the solvent is able to flow through as well as around the coil. Hence, the flow of an incompressible fluid through and around a macromolecule represented by a porous sphere may be solved from the Debije-Brinkman equation:

$$
-\nabla P+\eta_{0} \Delta V-\frac{\eta_{0}}{k}(\boldsymbol{V}-\boldsymbol{U})=0,
$$

$\operatorname{div} \boldsymbol{V}=0$.

In this equation, which applies to the stationary state only, $V$ and $P$ denote the average local velocity and pressure of the solvent, $U$ is the local velocity of the polymeric material, $\eta_{0}$ the viscosity of the pure solvent, and $k$ the local permeability.

It is implicit in the use of the Debije-Brinkman equation that the polymer is represented by a continuum with a locally varying permeability. This equation has been used by Ooms et al. $^{2}$ ) to calculate sedimentation coefficients; the values thus obtained agreed within a few percent with the experimental values. A microscopic derivation of an equation very similar to (1) was given 
by Felderhof and Deutch ${ }^{3}$ ); a macroscopic derivation of (1) with a discussion of the effects due to polymer solvent interaction was given by Wiegel and Mijnlieff ${ }^{4}$.

In a real macromolecule the permeability will be some function $k(r)$ of the radial distance $(r)$ to the centre of the coil. This function is not related in a simple way to the local segment density $[c(r)]$, because permeability appears to depend strongly on the quality of the solvent [see Mijnlieff and Jaspers $\left.{ }^{5}\right)$ ].

Although an a priori calculation of $k(r)$ is unfeasible, this function can be determined in the following way. First one uses an exact relation between the permeability and the sedimentation coefficient to determine the permeability at a certain concentration $[k(c)]$ from the sedimentation coefficient at the same concentration, which can be measured accurately [compare Mijnlieff and Jaspers ${ }^{5}$ ) and Wiegel and Mijnlieff $\left.{ }^{4}\right)$ ]. Next, one assumes a certain form for the function $c(r)$, after which on combining with $k(c)$ the function $k(r)$ is obtained. On adopting a gaussian for $c(r)$ one finds in this way that the radial dependence of the permeability is approximately described by

$$
k(r)=K \exp \left(Q r^{2}\right)
$$

where $K$ and $Q$ are positive constants the values of which depend on the temperature and on the nature of the polymer and solvent.

It is the aim of the present paper to calculate the intrinsic viscosity and the translational friction coefficient of macromolecules which are characterized by a local permeability given by the last equation. In the past (1) has been solved for a few specific choices of $k(r)$. Debije and Bueche ${ }^{6}$ ) solved (1) for a sphere of constant permeability, both for shear flow and for translation. Felderhof ${ }^{7}$ ) presented a very general formalism for the calculation of frictional properties of permeable macromolecules, but gave explicit results only for the uniform sphere and for the spherical shell. We shall use Felderhof's methods to study the more realistic case (3).

The content of the paper is divided as follows. Section 2 is devoted to the derivation of a general expression for the viscosity. In section 3 we specialize to the model characterized by eq. (3) and give the results of the numerical solution of the relevant system of differential equations. Similarly, section 4 is devoted to the derivation of a general expression for the translational friction coefficient and the results for the model characterized by eq. (3) are given in section 5. The comparison between predicted and measured values of the intrinsic viscosity and friction coefficient (which turns out to be satisfactory) and the relevance of these results for the understanding of frictional properties of polymers in solution are the subject of a separate paper ${ }^{8}$ ). (Also compare ref. 10 ).

\section{General expression for the viscosity}

Consider an isolated macromolecule which is located at the origin of a cartesian frame of coordinates. Due to the presence of the macromolecule in 
the origin the velocity and pressure will be the solutions of eqs. (1) and (2) which approach the simple shear flow given by

$$
v_{1}=+G_{\mathrm{e}} y ; \quad v_{2}=0 ; \quad v_{3}=0,
$$

at large distances from the origin.

The macromolecule is represented by a rigid porous sphere with a permeability $k(r)$ which has spherical symmetry, but which is otherwise arbitrary. Owing to the interaction with the fluid this sphere will start to rotate around the $z$-axis with an angular velocity $\omega$ :

$$
U_{1}=-\omega y ; \quad U_{2}=+\omega x ; \quad U_{3}=0 .
$$

In the stationary state the total torque of the forces which the fluid exerts on the coil should vanish. It can easily be shown that this implies that the angular velocity equals half the shear rate:

$$
\omega=-\frac{1}{2} G_{\mathrm{e}}
$$

For the actual velocity and pressure fields we make the Ansatz due to Felderhof ${ }^{7}$ ):

$$
\begin{aligned}
& V=U+\phi(v-U)-\mu r \times(r \times[v-U]), \\
& P=p_{0}-\eta_{0} \chi \frac{r \cdot v}{r^{2}}
\end{aligned}
$$

Here $\phi, \mu$ and $\chi$ are three unknown functions of the radial distance $(r)$ to the origin. It is straightforward to verify that (7) and (8) are indeed the solution of (1) and (2) provided

$$
\begin{aligned}
& \mu=\frac{1}{3 r} \phi^{\prime}, \\
& \phi^{\prime \prime}+\frac{6}{r} \phi^{\prime}-k^{-1} \phi+\frac{\chi^{\prime}}{r}=0, \\
& \chi^{\prime \prime}+\frac{2}{r} \chi^{\prime}-\frac{6}{r^{2}} \chi-r\left(k^{-1}\right)^{\prime} \phi=0 .
\end{aligned}
$$

The prime denotes differentiation with respect to $r$. The boundary conditions are that the velocity and pressure stay finite in the origin and approach the unperturbed fields $v(r)$ and $p_{0}$ at distances which are large compared to the diameter of the coil; this implies:

$$
\begin{aligned}
& \phi(0) \text { finite, } \\
& \chi(0) \text { finite, } \\
& \phi(\infty)=1, \\
& \chi(\infty)=0 .
\end{aligned}
$$


For $r \rightarrow \infty$ the permeability goes to infinity and the fourth term on the left-hand side of eq. (11) vanishes. The resulting equation has the solution

$$
\chi(r) \cong 2 A / r^{3} \quad(r \rightarrow \infty) .
$$

Upon substitution into eq. (10) one finds

$$
\phi(r) \cong 1-\frac{A}{r^{3}}+\frac{B}{r^{5}} \quad(r \rightarrow \infty),
$$

where the boundary conditions (12) were used and where the third term on the left-hand side of eq. (10) was set equal to zero. By substituting into (7) one finds the asymptotic form of the three components of the velocity:

$$
\begin{aligned}
& V_{1}=+G_{\mathrm{e}} y-G_{\mathrm{e}} A \frac{x^{2} y}{r^{5}}+\mathcal{O}\left(r^{-4}\right), \\
& V_{2}=-G_{\mathrm{e}} A \frac{x y^{2}}{r^{5}}+\mathcal{O}\left(r^{-4}\right), \\
& V_{3}=-G_{\mathrm{e}} A \frac{x y z}{r^{5}}+\mathcal{O}\left(r^{-4}\right),
\end{aligned}
$$

which formulae determine the viscosity in the following way. Consider a dilute solution which contains $n_{\mathrm{p}}$ of these macromolecules per unit volume. Let the solution be contained between two parallel walls situated at $y= \pm L$ and let these walls move with equal but opposite velocities ( $\pm G L$ ) along the $x$-axis in such a way that the macroscopic velocity field has a shear rate $G$. We calculate the viscosity of the solution following the method of Burgers ${ }^{9}$ ). The macroscopic velocity field (macroscopic shear rate $G$ ) should be distinguished from the local velocity field in the vicinity of a macromolecule. The velocity field which would be found at the position $\left(\boldsymbol{r}_{i}\right)$ of a particular macromolecule, when that molecule has first been removed, has a shear rate $G_{\mathrm{e}}$. The presence of the molecule at $\boldsymbol{r}_{i}$ will add to this unperturbed velocity a small correction given by (15), and these corrections have to be summed over all the coils in the fluid.

To be more specific we calculate the correction to the velocity in some point $(x, y, z)$ due to all coils present in a thin slice of fluid parallel to the $x, z$-plane and with thickness $\mathrm{d} y^{\prime}$. As the coils are distributed with number density $n_{\mathrm{p}}$ the correction to $V_{\mathrm{I}}$ can be found from the integral

$$
\begin{aligned}
\Delta V_{\mathrm{l}} & =-G_{\mathrm{e}} A n_{\mathrm{p}} \mathrm{d} y^{\prime} \int_{-\infty}^{+\infty} \mathrm{d} x^{\prime} \int_{-\infty}^{+\infty} \mathrm{d} z^{\prime} \frac{\left(x-x^{\prime}\right)^{2}\left(y-y^{\prime}\right)}{\left|\boldsymbol{r}-\boldsymbol{r}^{\prime}\right|^{5}} \\
& =-\frac{2}{3} \pi G_{\mathrm{e}} A n_{\mathrm{p}} \frac{y-y^{\prime}}{\left|y-y^{\prime}\right|} \mathrm{d} y^{\prime} .
\end{aligned}
$$

Note that $\partial \Delta V_{1} / \partial y=0$, so the molecules in this slice do not change the local shear rate; therefore the shear at the upper and lower plates still equals $G_{\mathrm{e}}$. 
Hence, the $x$-component of the force which the fluid exerts on a unit area of the upper surface equals $\eta_{0} G_{\mathrm{e}}$. By integrating (16) over $y^{\prime}$ and adding the local velocity field one finds the macroscopic velocity field $\boldsymbol{V}^{(\mathrm{M})}$ described by

$$
\begin{aligned}
& V_{1}^{(\mathrm{M})}=+G y, \\
& V_{2}^{(\mathrm{M})}=0, \\
& V_{3}^{(\mathrm{M})}=0 .
\end{aligned}
$$

Carrying out the integration one finds:

$$
G=\left(1-\frac{4}{3} \pi n_{\mathrm{p}} A\right) G_{\mathrm{e}} .
$$

The viscosity $\eta$ of the solution is operationally defined by measuring the force per unit area and dividing by the shear rate. But as the force per unit area has an $x$-component equal to $\eta_{0} G_{\mathrm{e}}$ this gives

$$
\eta G=\eta_{0} G_{\mathrm{e}}
$$

Combining the last two equations gives, for the relative increase of the viscosity,

$$
\left(\eta-\eta_{0}\right) / \eta_{0}=\frac{4}{3} \pi n_{\mathrm{p}} A .
$$

Together with eqs. (10)-(14) this relation expresses the viscosity of a dilute solution of permeable macromolecules in terms of the asymptotic behaviour of the flow field around one macromolecule. The result (20) was first obtained by Burgers ${ }^{9}$ ).

\section{Application to a realistic polymer model}

We now apply the general theory which formed the subject of section 2 to a realistic model for which the radial dependence of the permeability is given by (3). When this expression is substituted into (10) and (11) and the dimensionless distance $x=r \sqrt{Q}$ is introduced as the independent variable, the differential equations become

$$
\begin{aligned}
& \frac{\mathrm{d}^{2} f}{\mathrm{~d} x^{2}}+\frac{6}{x} \frac{\mathrm{d} f}{\mathrm{~d} x}-\alpha \mathrm{e}^{-x^{2}} f+\frac{1}{x} \frac{\mathrm{d} g}{\mathrm{~d} x}=0, \\
& \frac{\mathrm{d}^{2} g}{\mathrm{~d} x^{2}}+\frac{2}{x} \frac{\mathrm{d} g}{\mathrm{~d} x}-\frac{6}{x^{2}} g+2 \alpha x^{2} \mathrm{e}^{-x^{2}} f=0 .
\end{aligned}
$$

Here $f(x)=\phi(r), g(x)=\chi(r)$, and $\alpha$ denotes the dimensionless parameter

$$
\alpha=(K Q)^{-1} \text {. }
$$

The boundary conditions on $f$ and $g$ are the same as those on $\phi$ and $\chi$.

Whereas an analytic solution of (21) seems difficult to obtain, a numerical solution can be found, for example by a process of iteration. Note that $g$ can be calculated from $f$ by solving $(21 \mathrm{~b})$ : 


$$
g(x)=\sum_{\xi}^{2} \alpha x^{-3} \int_{0}^{1} y^{h} \mathrm{e}^{-y^{2}} f(y) \mathrm{d} y+\frac{2}{5} \alpha x^{2} \int_{x}^{x} y \mathrm{e}^{-y^{2}} f(y) \mathrm{d} y .
$$

On the other hand, once the function

$$
F(x)=+\alpha \mathrm{e}^{-x^{2}} f(x)-\frac{1}{x} \frac{\mathrm{d} g}{\mathrm{~d} x}
$$

is known. (21a) can be solved for $f$ :

$$
f(x)=1-\frac{1}{5} x^{-5} \int_{0}^{x} y^{6} F(y) \mathrm{d} y-\frac{1}{5} \int_{x}^{x} y F(y) \mathrm{d} y .
$$

The numerical procedure starts with some guess for the function $f(x)$. Substitution into (23) gives a guess for $g(x)$ and hence for $F(x)$ by substitution into (24). When this approximation of $F(x)$ is substituted into (25) one finds an improved approximation of $f(x)$. The iteration is continued till the successive approximations differ by an amount which is smaller than some error margin. Note that (23) immediately leads to the asymptotic behaviour of $g(x)$; using (13) and (20) this gives for the relative increase of the viscosity

$$
\frac{\eta-\eta_{0}}{\eta_{0}}=\frac{4}{3} \pi n_{\mathrm{p}} K^{1} Q^{-5 / 2} \Phi\left(K^{-1} Q^{-1}\right)
$$

where

$$
\Phi(\alpha)=\frac{1}{5} \int_{0}^{\infty} x^{6} \mathrm{e}^{-x^{2}} f(x) \mathrm{d} x .
$$

TABLE I

The function $\Phi$. defined by eq. (26b), and the value of $f(x)$ in the origin

\begin{tabular}{llllll}
\hline$\alpha$ & $\Phi(\alpha)$ & $f(0)$ & $\alpha$ & $\Phi(\alpha)$ & $f(0)$ \\
\hline 0.0 & 0.332 & 1.000 & 2.0 & 0.311 & 0.825 \\
0.1 & 0.331 & 0.990 & 3.0 & 0.301 & 0.754 \\
0.2 & 0.330 & 0.980 & 4.0 & 0.292 & 0.692 \\
0.3 & 0.329 & 0.971 & 5.0 & 0.284 & 0.637 \\
0.4 & 0.328 & 0.961 & 6.0 & 0.277 & 0.588 \\
0.5 & 0.327 & 0.952 & 7.0 & 0.270 & 0.544 \\
0.6 & 0.325 & 0.942 & 8.0 & 0.263 & 0.505 \\
0.7 & 0.324 & 0.933 & 9.0 & 0.257 & 0.470 \\
0.8 & 0.323 & 0.924 & 10 & 0.251 & 0.439 \\
0.9 & 0.322 & 0.915 & 11 & 0.246 & 0.411 \\
1.0 & 0.321 & 0.907 & 12 & 0.241 & 0.385 \\
1.1 & 0.320 & 0.898 & 13 & 0.236 & 0.362 \\
1.2 & 0.319 & 0.889 & 14 & 0.231 & 0.341 \\
1.3 & 0.318 & 0.881 & 15 & 0.227 & 0.321 \\
1.4 & 0.317 & 0.873 & 16 & 0.223 & 0.304 \\
1.5 & 0.316 & 0.865 & 17 & 0.219 & 0.288 \\
& & & 18 & 0.215 & 0.273 \\
\hline
\end{tabular}




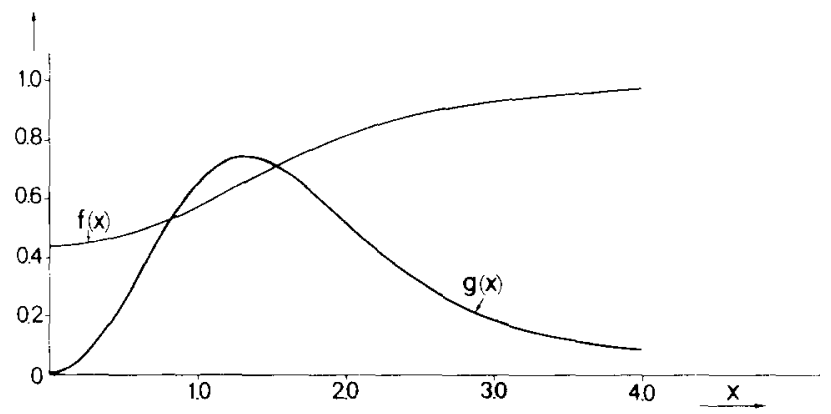

Fig. 1. Behaviour of the functions $f(x)$ and $g(x)$ for $\alpha=10$.

This function has been calculated numerically for values of $\alpha$ up to 18 . The results are collected in table I. Note that $\Phi(0)=\frac{3}{16} \sqrt{\pi}$. The behaviour of $f(x)$ and $g(x)$ is drawn in fig. 1 for the typical value $\alpha=10$. If $m$ denotes the mass of a single macromolecule the intrinsic viscosity $[\eta]$ is given by

$$
[\eta]=\frac{4}{3} \pi m^{-1} K^{-1} Q^{-5 / 2} \Phi\left(K^{-1} Q^{-1}\right) .
$$

Together with the numerical results in table I this formula enables us to predict the intrinsic viscosity of dilute polymer solutions. This is the subject of a separate paper ${ }^{8}$ ). (Also compare ref. 10).

Table I also gives the value $f(0)$ of $f(x)$ in the origin of the coil, which is a measure for the degree of draining of the coil. The velocity on the $y$-axis is found by substituting $x=z=0$ into (7):

$V_{\mathrm{I}}=\frac{1}{2} G_{\mathrm{e}} y(1+\phi)+\frac{1}{2} G_{\mathrm{e}} y^{3} \frac{1}{3 r} \frac{\mathrm{d} \phi}{\mathrm{d} r}$,

where $x=r_{1}, y=r_{2}, z=r_{3}$. This velocity has been drawn in fig. 2 for $\alpha=10$.

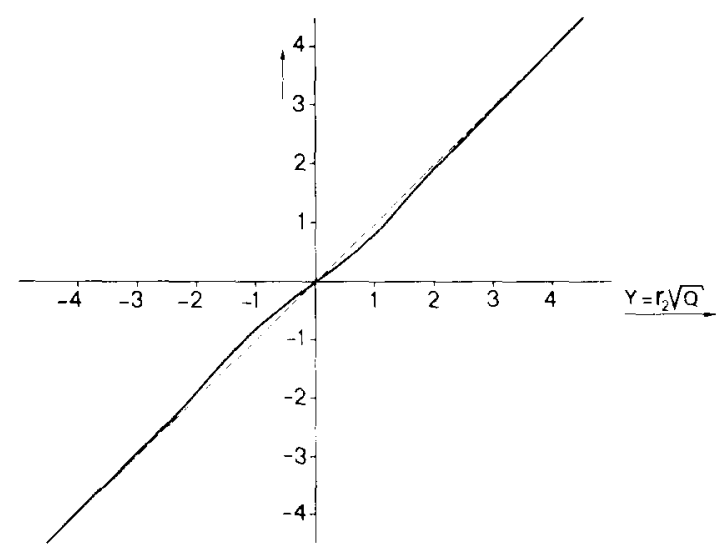

Fig. 2. The velocity component $V_{1}$ in the $r_{1}$ direction as a function of $r_{2} \sqrt{Q}$ for $\alpha=10$. The vertical coordinate measures the value of $V_{1} Q^{1 / 2} G_{e}^{-1}$. 


\section{General expression for the friction coefficient}

When a hard sphere of radius $R$ moves through a viscous fluid with a constant relative velocity $v_{0}$, the force on the sphere is given by Stokes' formula

$$
F=-6 \pi \eta_{0} R v_{0},
$$

and the friction coefficient $f$ is given by the ratio of force and velocity:

$$
f=\left|F / v_{0}\right|=6 \pi \eta_{0} R .
$$

These formulae are often used to assign an effective hydrodynamic radius to a macromolecule of which $f$ has been measured. However, the same criticism applies here as in the case of the intrinsic velocity, and in this section we calculate the frictional coefficient from the Debije-Brinkman equation (1), where now $\boldsymbol{U}=0$.

Consider an isolated macromolecule located at the origin of a cartesian system of coordinates. In the absence of the molecule the fluid would be in a state of uniform flow and the velocity $v$ has the components

$$
v_{1}=0 ; \quad v_{2}=0 ; \quad v_{3}=+v_{0} .
$$

The pressure would equal a constant $p_{0}$ everywhere in the fluid. Owing to the presence of the macromolecular coil in the origin the actual velocity and pressure will be the solutions of (1) and (2) which approach the unperturbed fields at large distances from the origin.

The macromolecule is again represented by a rigid porous sphere with a permeability $k(r)$ which has spherical symmetry, but which is otherwise arbitrary. For the actual velocity field and pressure field we use the Ansatz due to Felderhof ${ }^{7}$ ):

$$
\begin{aligned}
& \boldsymbol{V}=\psi(\boldsymbol{r}) \boldsymbol{v}-\nu(\boldsymbol{r}) \boldsymbol{r} \times(\boldsymbol{r} \times \boldsymbol{v}), \\
& P=p_{0}-\eta_{0} \xi(r) \frac{\boldsymbol{r} \cdot \boldsymbol{v}}{r}
\end{aligned}
$$

Here $\psi, \nu$ and $\xi$ are three unknown functions of the radial distance to the origin. It is straightforward to verify that (32) solves (1) and (2) provided

$$
\begin{aligned}
& \nu=\frac{1}{2 r} \psi^{\prime}, \\
& \psi^{\prime \prime}+\frac{4}{r} \psi^{\prime}-k^{-1} \psi+\xi^{\prime}=0, \\
& \xi^{\prime \prime}+\frac{2}{r} \xi^{\prime}-\frac{2}{r^{2}} \xi-\left(k^{-1}\right)^{\prime} \psi=0
\end{aligned}
$$

The prime denotes differentiation with respect to $r$. The boundary conditions are that the velocity and pressure stay finite in the origin and approach the 
unperturbed fields at large $r$; this implies

$$
\begin{aligned}
& \psi(0) \text { finite, } \\
& \xi(0) \text { finite, } \\
& \psi(\infty)=1, \\
& \psi(\infty)=0 .
\end{aligned}
$$

For $r \rightarrow \infty$ the function $k^{-1}$ vanishes and the last term on the left-hand side of (33c) vanishes. The resulting equation has the solution

$$
\xi(r) \cong C / r^{2} \quad(r \rightarrow \infty),
$$

where the boundary condition (34d) was used. Substituting into (33b) and using the boundary condition (34c) one finds the asymptotic behaviour

$$
\psi(r) \cong 1-\frac{C}{r}+\frac{D}{r^{3}} \quad(r \rightarrow \infty) .
$$

These asymptotic formulae determine the friction coefficient in the following way. From the macroscopic derivation of the Debije-Brinkman equation, given for example by Wiegel and Mijnlieff ${ }^{4}$ ), it is clear that the $z$-component of the total force which the fluid exerts on the coil equals

$$
F_{3}=\eta_{0} \int k^{-1}(\boldsymbol{r}) V_{3}(\boldsymbol{r}) \mathrm{d}^{3} \boldsymbol{r} \text {. }
$$

Using (1) this can be written in the form

$$
F_{3}=\int\left(-\frac{\partial P}{\partial z}+\eta_{0} \Delta V_{3}\right) \mathrm{d}^{3} \boldsymbol{r} .
$$

With Gauss's theorem the volume integral can be replaced by a surface integral over the surface of a sphere with a large radius $r$; subsequently the limit $r \rightarrow \infty$ will be taken. Denoting the surface element of this sphere by $\mathrm{d}^{2} S$ one finds

$$
F_{3}=-\oint P \frac{z}{r} \mathrm{~d}^{2} S+\eta_{0} \oint\left(\frac{\partial V_{3}}{\partial x} \frac{x}{r}+\frac{\partial V_{3}}{\partial y} \frac{y}{r}+\frac{\partial V_{3}}{\partial z} \frac{z}{r}\right) \mathrm{d}^{2} S .
$$

The advantage of this approach is that only the asymptotic form of $V_{3}$ and $P$ is needed.

Using the results (32), (33), (35) and (36) one finds

$$
\begin{aligned}
& V_{3} \cong v_{0}-\frac{v_{0} C}{2 r}-\frac{v_{0} C z^{2}}{2 r^{3}}+\mathcal{O}\left(r^{-3}\right), \\
& P \cong p_{0}-\eta_{0} \frac{v_{0} C z}{r^{3}}
\end{aligned}
$$

Substituting this into (38) and taking the limit $r \rightarrow \infty$ one finds

$$
F_{3}=4 \pi \eta_{0} C v_{0} \text {. }
$$


Hence by $(30)$ the friction coefficient is

$$
f=4 \pi \eta_{0} C
$$

its value depends only on the value of the constant $C$, i.e. on the leading term in the asymptotic behaviour of pressure and velocity.

\section{Application to a realistic polymer model}

We now apply the general theory which formed the subject of section 4 to the model for which $k(r)$ is given by eq. (3). When this expression is substituted into (33b) and (33c) and when the dimensionless distance $x=r \sqrt{Q}$ is introduced as the independent variable, the differential equations become

$$
\begin{aligned}
& \frac{\mathrm{d}^{2} h}{\mathrm{~d} x^{2}}+\frac{4}{x} \frac{\mathrm{d} h}{\mathrm{~d} x}-\alpha \mathrm{e}^{-x^{2}} h+\frac{\mathrm{d} q}{\mathrm{~d} x}=0, \\
& \frac{\mathrm{d}^{2} q}{\mathrm{~d} x^{2}}+\frac{2}{x} \frac{\mathrm{d} q}{\mathrm{~d} x}-\frac{2}{x^{2}} q+2 \alpha x \mathrm{e}^{-x 2} h=0 .
\end{aligned}
$$

Here $h(x)=\psi(r)$ and $q(x)=Q^{-1 / 2} \xi(r)$. The boundary conditions on $h$ and $q$ are the same as those on $\psi$ and $\xi$. It is straightforward to show that the boundary condition that $h$ and $q$ should be finite in the origin, is equivalent to the boundary conditions

$$
\begin{aligned}
& q(0)=0, \\
& h^{\prime}(0)=0,
\end{aligned}
$$

which are easier to use.

Whereas an analytic solution of (42) seems difficult to obtain, a numerical solution can be found in the following way. We set

$$
h(x)=1-\frac{c}{x}+\frac{d}{x^{3}}, \quad q(x)=\frac{c}{x^{2}} \quad\left(x>x_{0}\right),
$$

for $x$ larger than some large positive constant $x_{0}$, then use (42) to calculate $q(0)$ and $h^{\prime}(0)$. In general these values will violate the boundary conditions (43). The values of $c$ and $d$ are then adjusted till (43) is fulfilled within some error margin. Denoting the correct value of $c$ by $\alpha \Psi(\alpha)$ we have

$$
C=Q^{-1 / 2} c=Q^{-1 / 2} \alpha \Psi(\alpha) .
$$

For the record only we just mention that it can easily be shown that

$$
\Psi(\alpha)=\frac{2}{3} \int_{0}^{\infty} x^{4} \mathrm{e}^{-x^{2}} f(x) \mathrm{d} x .
$$

This implies, among others, that

$$
\Psi(0)=\frac{2}{3} \int_{0}^{\infty} x^{4} \mathrm{e}^{-x^{2}} \mathrm{~d} x=\frac{1}{4} \sqrt{\pi} .
$$


TABLE II

The function $\Psi$, defined by eq. (46)

\begin{tabular}{llllllll}
\hline$\alpha$ & $\Psi(\alpha)$ & $\alpha$ & $\Psi(\alpha)$ & $\alpha$ & $\Psi(\alpha)$ & $\alpha$ & $\Psi(\alpha)$ \\
\hline 0 & 0.443 & 5 & 0.209 & 10 & 0.141 & 15 & 0.108 \\
1 & 0.359 & 6 & 0.190 & 11 & 0.133 & 16 & 0.103 \\
2 & 0.303 & 7 & 0.175 & 12 & 0.125 & 17 & 0.099 \\
3 & 0.263 & 8 & 0.162 & 13 & 0.119 & & \\
4 & 0.233 & 9 & 0.151 & 14 & 0.113 & & \\
\hline
\end{tabular}

The friction coefficient follows upon substitution of (45) into (41):

$$
f=4 \pi \eta_{0} Q^{-1 / 2} \alpha \Psi(\alpha) .
$$

The values of the function $\Psi(\alpha)$ have been calculated for values of $\alpha$ up to 17; the results are collected in table II. The behaviour of $h(x)$ and $q(x)$ is drawn in figs. 3 and 4 for some typical values of $\alpha$. For a comparison between the predicted and measured values of the friction coefficient we again refer to Mijnlieff and $\mathrm{Wiegel}^{8}$ ).

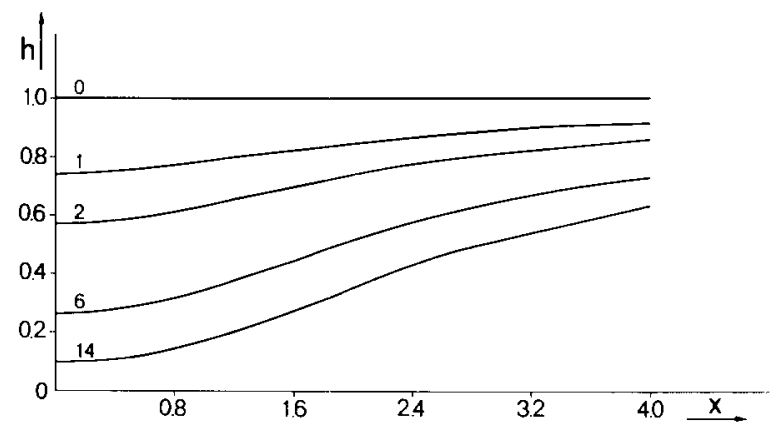

Fig. 3. The function $h(x)$ for $\alpha=0,1,2,6$ and 14 .

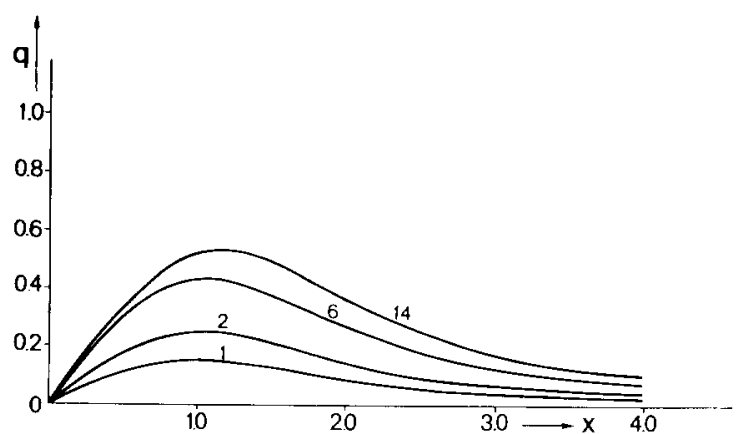

Fig. 4. The function $q(x)$ for $\alpha=0,1,2,6$ and 14 . 
The velocity in the $z$-direction is given by

$$
V_{3}=\psi v_{0}+\left(x^{2}+y^{2}\right) \frac{v_{0}}{2 r} \frac{\mathrm{d} \psi}{\mathrm{d} r}
$$

where $x=r_{1}, y=r_{2}$, and $z=r_{3}$. Hence, along the $z$-axis the velocity is given by $V_{3}(0,0, z)=\psi v_{0}$; this profile is essentially given in fig. 3 . Along the $x$-axis the $z$-component of the velocity equals

$$
V_{3}(x, 0,0)=\psi v_{0}+x^{2} \frac{v_{0}}{2 r} \frac{\mathrm{d} \psi}{\mathrm{d} r}
$$

this profile is drawn in fig. 5 for $\alpha=14$.

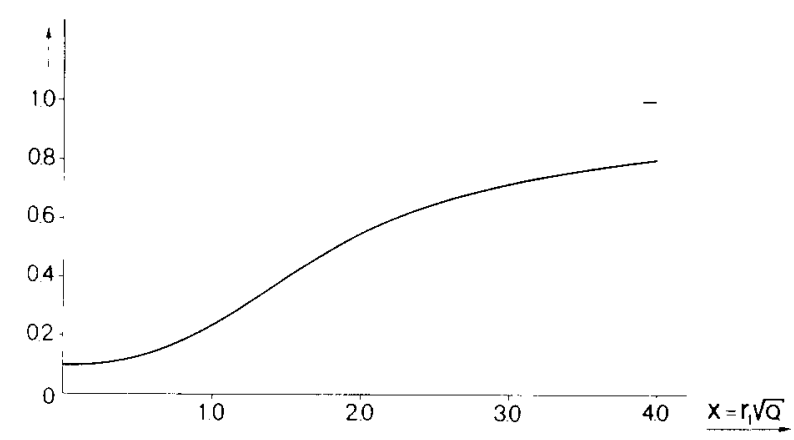

Fig. 5. The velocity component $V_{3}$ as a function of $r_{1} \sqrt{Q}$ for $\alpha=14$. The vertical coordinate measures the value of $V_{3} v_{0}^{-1}$. The limiting value 1 is indicated by a short horizontal bar.

\section{References}

1) A. Einstein, Ann. der Phys. 19 (1906) 289; corrections in Ann. der Phys. 34 (1911) 591. Reprinted in Investigations on the Theory of the Brownian Movement (Dover, New York. 1956) pp. 36-62.

2) G. Ooms, P.F. Mijnlieff and H.L. Beckers, J. Chem. Phys. 53 (1970) 4123.

3) B.U. Felderhof and J.M. Deutch, J. Chem. Phys. 62 (1975) 2391, 2398.

4) F.W. Wiegel and P.F. Mijnlieff, Physica 85A (1976) 207.

5) P.F. Mijnlieff and W.J.M. Jaspers, Trans. Faraday Soc. 67 (1971) 1837.

6) P. Debije and A.M. Bueche, J. Chem. Phys. 16 (1948) 573.

7) B.U. Felderhof, Physica 80A (1975) 63, 172

8) P.F. Mijnlieff and F.W. Wiegel, to appear.

9) J.M. Burgers, Proc. Roy. Acad. Sci. Amsterdam (I) 16 (1) (1938) 128.

10) F.W. Wiegel and P.F. Mijnlieff, Polymer 18 (1977) 636. 